\title{
EITC is Vital for Working-Poor Families in Rural America
}

\author{
WILLIAM O'HARE AND ELIZABETH KNEEBONE
}

I n Tax Year 2004, tax filers claimed almost $\$ 40$ billion through the Earned Income Tax Credit (EITC), making the EITC one of the largest federal programs that provides cash supports to low-income working families in the United States. This Fact Sheet examines rural/urban differences in the Federal EITC program. ${ }^{1}$

The EITC, a refundable tax credit, acts as a wage supplement and work incentive for low-income workers. Unlike many other means-tested support programs, the EITC does not require a separate bureaucracy to administer benefits because it works through the tax code. To claim the credit, individuals must work (have earned income) and file a tax return. The vast majority of EITC dollars go to working families with children who have earnings below $\$ 35,000$ a year. The low administrative cost, built-in work incentive, and focus on families with children make the EITC appealing to legislators across the political spectrum.

The EITC is especially important to rural families throughout the United States. Among poor and near-poor families, those in rural areas are more likely to be working, and they are more likely to be working in low-wage jobs. Almost half (48 percent) of rural children live in low-income families (incomes less that twice the poverty line) compared with 37 percent of urban children. ${ }^{2}$ These factors contribute to the higher rate of EITC receipt in rural areas across the country, and underline the importance of the benefit to these families. Receipt of EITC in rural areas also has been boosted in recent years by programs designed to make sure all those families who are eligible get this benefit. ${ }^{3}$

While only 16 percent of U.S. tax filers in 2004 were from rural areas, rural EITC filers claimed 20 percent of the 39.8 billion EITC dollars. Rural EITC recipients collectively received $\$ 7.8$ billion through the credit in 2004 .

The amount of money received by the average rural family $(\$ 1,850)$ may not seem like much to more affluent families, but for low-income families it can be an enormous help. For kids growing up in low-income families, even a small boost in income can lead to better child outcomes; a few thousand dollars a year can have significant impacts. ${ }^{4}$

As Congress entertains legislation to expand the EITC program, it is important to recognize how vital this program is for low-income families in rural America. For a large segment of rural families, and upwards of one-third of families in some states in the rural South, the EITC provides an important source of income that can supplement stagnant wages, help a family hold onto a home, repair a car to keep working, or cover health bills.

Several key facts are clear:

- Nationwide a higher percentage of rural tax filers (20 percent) receives the EITC than urban filers (16 percent). And the same is true in almost every state. Rural tax filers receive the EITC at a higher rate than urban filers in 42 of 48 states (All counties in New Jersey and Rhode Island are in Metropolitan Areas).

- The rate of rural EITC receipt varies greatly depending on the state. The states where receipt of EITC among rural families is the highest are in the South and Southwest. Mississippi, Louisiana, and Georgia each had more than 30 percent of their rural tax filers receive the credit in 2004.

- States in the South and Southwest are where the rural/urban gap in the percentage of fillers receiving EITC is largest. Leading the list, rural tax filers in Georgia and Arizona are much more likely to receive the EITC than their urban counterparts.

- At the national level, there is little difference in the average amount of ETIC received -- $\$ 1,831$ for urban families compared to $\$ 1,850$ for rural families. But there are substantial differences across the rural areas of states, ranging from a low of $\$ 1,232$ in Massachusetts to a high of \$2,193 in Louisiana.

- Most of the states where rural families receive a relatively high level of EITCs are located in the South: Louisiana $(\$ 2,193)$, Mississippi $(\$ 2,183)$, Georgia, $(\$ 2,099)$, Alabama $(\$ 2,083)$, and Texas $(\$ 2,048)$. The states with lower average credits for rural families are almost all located in New England: Massachusetts, Alaska, Vermont, New Hampshire, and Connecticut.

\section{A U T H O R S}

Dr. William O'Hare is a Rural Fellow at the Carsey Institute. (bbohare@comcast.net)

Elizabeth KneEbone is a Research Analyst at the Brookings Institution's Metropolitan Policy Program. (EKneebone@brookings.edu)

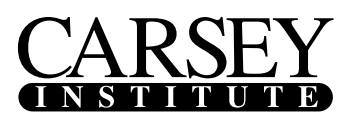

Building KNOWLEDge for FAMilies AND Communities in the 21St Century
Huddleston Hall, 73 Main Street, Durham, NH 03824

(603) 862-2821 • www.carseyinstitute.unh.edu

The Carsey Institute Reports on Rural America are supported by the Annie E. Casey Foundation's initiative to strengthen rural families, the Ford Foundation, and the W.K. Kellogg Foundation. 
Table 1. Receipt of EITC in RuRal and Urban Households: Tax Year 2004

\begin{tabular}{|c|c|c|c|c|c|c|c|}
\hline \multirow[b]{2}{*}{ State } & \multicolumn{3}{|c|}{ RURAL (Outside Metropolitan Areas) } & \multicolumn{3}{|c|}{ URBAN (Inside Metropolitan Areas) } & \multirow{2}{*}{$\begin{array}{l}\text { Rural/Urban } \\
\text { Percentage-poin } \\
\text { Difference in } \\
\text { Receiving ElTC }\end{array}$} \\
\hline & $\begin{array}{l}\text { Share Receiving } \\
\text { EITC }\end{array}$ & $\begin{array}{c}\text { EITC Amount } \\
\text { (\$ in millions) }\end{array}$ & $\begin{array}{c}\text { Average EITC } \\
\text { Amount }\end{array}$ & $\begin{array}{l}\text { Share } \\
\text { EITC }\end{array}$ & $\begin{array}{c}\text { EITC Amount } \\
\text { (\$ in millions) }\end{array}$ & $\begin{array}{c}\text { Average EITC } \\
\text { Amount }\end{array}$ & \\
\hline US & $20.4 \%$ & $7,835.2$ & $\$ 1,850$ & $16.3 \%$ & $31,926.9$ & $\$ 1,831$ & $4.1 \%$ \\
\hline Alabama & $29.2 \%$ & 316.4 & $\$ 2,083$ & $24.6 \%$ & 694.7 & $\$ 2,109$ & $4.6 \%$ \\
\hline Alaska & $13.9 \%$ & 22.9 & $\$ 1,516$ & $10.6 \%$ & 37.0 & $\$ 1,568$ & $3.3 \%$ \\
\hline Arizona & $25.9 \%$ & 115.5 & $\$ 1,936$ & $16.2 \%$ & 605.8 & $\$ 1,817$ & $9.7 \%$ \\
\hline Arkansas & $28.4 \%$ & 246.4 & $\$ 1,977$ & $22.6 \%$ & 292.6 & $\$ 1,937$ & $5.9 \%$ \\
\hline California & $15.7 \%$ & 84.7 & $\$ 1,602$ & $16.3 \%$ & $4,185.9$ & $\$ 1,801$ & $-0.6 \%$ \\
\hline Colorado & $15.5 \%$ & 69.3 & $\$ 1,629$ & $12.3 \%$ & 357.4 & $\$ 1,648$ & $3.2 \%$ \\
\hline Connecticut & $9.6 \%$ & 21.5 & $\$ 1,565$ & $10.2 \%$ & 246.2 & $\$ 1,627$ & $-0.6 \%$ \\
\hline$D C$ & & & & $18.0 \%$ & 83.3 & $\$ 1,741$ & NA \\
\hline Delaware & $17.4 \%$ & 25.5 & $\$ 1,825$ & $13.9 \%$ & 74.2 & $\$ 1,750$ & $3.5 \%$ \\
\hline Florida & $21.9 \%$ & 178.8 & $\$ 1,860$ & $19.7 \%$ & $2,684.9$ & $\$ 1,839$ & $2.2 \%$ \\
\hline Georgia & $31.0 \%$ & 434.3 & $\$ 2,099$ & $21.0 \%$ & $1,270.9$ & $\$ 2,000$ & $10.0 \%$ \\
\hline Hawaii & $16.2 \%$ & 42.0 & $\$ 1,569$ & $13.8 \%$ & 95.3 & $\$ 1,631$ & $2.4 \%$ \\
\hline Idaho & $18.8 \%$ & 65.6 & $\$ 1,746$ & $16.4 \%$ & 108.6 & $\$ 1,747$ & $2.4 \%$ \\
\hline Illinois & $16.0 \%$ & 201.5 & $\$ 1,690$ & $14.9 \%$ & $1,344.2$ & $\$ 1,858$ & $1.1 \%$ \\
\hline Indiana & $15.3 \%$ & 160.1 & $\$ 1,688$ & $15.2 \%$ & 585.7 & $\$ 1,775$ & $0.1 \%$ \\
\hline lowa & $13.8 \%$ & 132.1 & $\$ 1,627$ & $12.1 \%$ & 142.2 & $\$ 1,633$ & $1.7 \%$ \\
\hline Kansas & $17.1 \%$ & 130.3 & $\$ 1,747$ & $13.0 \%$ & 168.4 & $\$ 1,709$ & $4.1 \%$ \\
\hline Kentucky & $24.1 \%$ & 292.8 & $\$ 1,804$ & $16.8 \%$ & 306.8 & $\$ 1,751$ & $7.3 \%$ \\
\hline Louisiana & $32.6 \%$ & 305.0 & $\$ 2,193$ & $27.7 \%$ & 796.7 & $\$ 2,149$ & $4.9 \%$ \\
\hline Maine & $16.5 \%$ & 66.7 & $\$ 1,623$ & $12.2 \%$ & 69.0 & $\$ 1,563$ & $4.3 \%$ \\
\hline Maryland & $14.5 \%$ & 33.8 & $\$ 1,720$ & $13.2 \%$ & 549.8 & $\$ 1,731$ & $1.3 \%$ \\
\hline Massachusetts & $8.4 \%$ & 1.5 & $\$ 1,232$ & $10.2 \%$ & 481.2 & $\$ 1,590$ & $-1.8 \%$ \\
\hline Michigan & $15.3 \%$ & 210.1 & $\$ 1,673$ & $14.3 \%$ & 930.1 & $\$ 1,809$ & $1.1 \%$ \\
\hline Minnesota & $13.6 \%$ & 139.2 & $\$ 1,604$ & $9.8 \%$ & 263.4 & $\$ 1,563$ & $3.8 \%$ \\
\hline Mississippi & $36.3 \%$ & 491.8 & $\$ 2,183$ & $27.7 \%$ & 295.6 & $\$ 2,088$ & $8.6 \%$ \\
\hline Missouri & $21.6 \%$ & 246.2 & $\$ 1,797$ & $15.6 \%$ & 524.4 & $\$ 1,777$ & $6.0 \%$ \\
\hline Montana & $17.5 \%$ & 81.5 & $\$ 1,693$ & $15.7 \%$ & 38.6 & $\$ 1,599$ & $1.8 \%$ \\
\hline Nebraska & $15.5 \%$ & 89.6 & $\$ 1,721$ & $12.3 \%$ & 95.0 & $\$ 1,693$ & $3.2 \%$ \\
\hline Nevada & $13.9 \%$ & 25.5 & $\$ 1,689$ & $15.2 \%$ & 244.3 & $\$ 1,710$ & $-1.3 \%$ \\
\hline New Hampshire & $11.3 \%$ & 42.3 & $\$ 1,564$ & $8.8 \%$ & 53.1 & $\$ 1,528$ & $2.5 \%$ \\
\hline New Jersey & & & & $12.0 \%$ & 838.4 & $\$ 1,743$ & NA \\
\hline New Mexico & $29.2 \%$ & 145.8 & $\$ 1,893$ & $21.8 \%$ & 213.1 & $\$ 1,812$ & $7.4 \%$ \\
\hline New York & $17.2 \%$ & 195.7 & $\$ 1,723$ & $17.3 \%$ & $2,402.5$ & $\$ 1,799$ & $-0.1 \%$ \\
\hline North Carolina & $24.7 \%$ & 519.1 & $\$ 1,935$ & $18.6 \%$ & 891.4 & $\$ 1,862$ & $6.0 \%$ \\
\hline North Dakota & $14.9 \%$ & 39.6 & $\$ 1,670$ & $10.9 \%$ & 23.9 & $\$ 1,553$ & $4.0 \%$ \\
\hline Ohio & $15.5 \%$ & 270.2 & $\$ 1,727$ & $14.4 \%$ & $1,110.6$ & $\$ 1,778$ & $1.1 \%$ \\
\hline Oklahoma & $24.3 \%$ & 230.7 & $\$ 1,887$ & $20.0 \%$ & 342.3 & $\$ 1,853$ & $4.3 \%$ \\
\hline Oregon & $17.6 \%$ & 102.6 & $\$ 1,709$ & $13.3 \%$ & 259.8 & $\$ 1,627$ & $4.4 \%$ \\
\hline Pennsylvania & $14.6 \%$ & 207.6 & $\$ 1,621$ & $13.2 \%$ & $1,076.4$ & $\$ 1,689$ & $1.4 \%$ \\
\hline Rhode Island & & & & $13.2 \%$ & 110.7 & $\$ 1,721$ & NA \\
\hline South Carolina & $29.7 \%$ & 251.2 & $\$ 2,004$ & $21.6 \%$ & 563.1 & $\$ 1,896$ & $8.0 \%$ \\
\hline South Dakota & $17.0 \%$ & 55.3 & $\$ 1,706$ & $13.4 \%$ & 35.6 & $\$ 1,612$ & $3.6 \%$ \\
\hline Tennessee & $23.7 \%$ & 284.2 & $\$ 1,823$ & $20.4 \%$ & 727.1 & $\$ 1,883$ & $3.4 \%$ \\
\hline Texas & $27.5 \%$ & 641.6 & $\$ 2,048$ & $23.2 \%$ & $3,807.2$ & $\$ 2,051$ & $4.3 \%$ \\
\hline Utah & $18.1 \%$ & 34.3 & $\$ 1,837$ & $13.8 \%$ & 207.5 & $\$ 1,741$ & $4.3 \%$ \\
\hline Vermont & $13.7 \%$ & 42.1 & $\$ 1,517$ & $10.1 \%$ & 14.6 & $\$ 1,448$ & $3.6 \%$ \\
\hline Virginia & $20.5 \%$ & 162.0 & $\$ 1,767$ & $13.4 \%$ & 695.4 & $\$ 1,765$ & $7.0 \%$ \\
\hline Washington & $15.8 \%$ & 87.7 & $\$ 1,720$ & $12.2 \%$ & 496.2 & $\$ 1,659$ & $3.6 \%$ \\
\hline West Virginia & $22.2 \%$ & 120.5 & $\$ 1,736$ & $17.4 \%$ & 125.1 & $\$ 1,684$ & $4.7 \%$ \\
\hline Wisconsin & $12.2 \%$ & 135.8 & $\$ 1,575$ & $11.0 \%$ & 343.5 & $\$ 1,665$ & $1.1 \%$ \\
\hline Wyoming & $13.5 \%$ & 36.0 & $\$ 1,615$ & $14.7 \%$ & 17.7 & $\$ 1,644$ & $-1.2 \%$ \\
\hline
\end{tabular}

Source: Brookings Institution analysis of Internal Revenue Service data

\section{E N D N O T E S}

1 "Rural" refers to nonmetropolitan counties and "urban" refers to counties inside metropolitan areas, as classified by the U.S. Office of Management and Budget.

${ }^{2}$ U.S. Census Bureau, available online at www.census.gov/hhes/www/poverty/detailedpovtabs.html
${ }^{3}$ For example, see The Annie E Casey Foundation, www.aecf.org/upload/ PDFFiles/FES/fes_eitc.pdf.

${ }^{4}$ Duncan, Greg J., Jeanne Brooks-Gunn, W. Jean Yeung, and Judith R. Smith, June 1998, "How Much Does Childhood Poverty Affect the Life Chances of Children?" American Sociological Review, Vol. 63, (pp 406-423). 